\title{
Exploration of the mechanism underlying the tumor necrosis avidity of hypericin
}

\author{
MARIE VAN DE PUTTE ${ }^{1}$, YICHENG NI $^{2}$ and PETER A.M. DE WITTE ${ }^{1}$
}

\author{
${ }^{1}$ Laboratory for Pharmaceutical Biology, Faculty of Pharmaceutical Sciences, K.U. \\ Leuven O and N II, Herestraat 49, Leuven; ${ }^{2}$ Department of Radiology, University Hospital, \\ Faculty of Medicine, K.U. Leuven University Hospital, Herestraat 49, 3000 Leuven, Belgium
}

Received September 13, 2007; Accepted October 30, 2007

\begin{abstract}
Hypericin, a potent necrosis avid agent, features a peculiar affinity for necrotic tissue. Necrosis avid contrast agents have been investigated as markers for non-invasive imaging of different disorders. In view of the promising clinical applications, a more complete knowledge of the mechanism of action is important for the future development of new chemical structures with improved characteristics. To study whether a compound-specific or non-specific mechanism based on plasma lipoprotein transport is involved in the accumulation of hypericin in intratumoral necrosis, we performed a visual and quantitative fluoromicroscopic analysis of the colocalization of hypericin and $\mathrm{DiOC}_{18}$-labeled lipoproteins in subcutaneous murine radiation-induced fibrosarcoma tumors. Microscopic fluorescent overlay images of necrotic tumors demonstrated that hypericin already showed clear necrosis avid characteristics $4 \mathrm{~h}$ after injection, whereas a similar outstanding accumulation in necrosis was not demonstrated for the labeled lipoproteins. Moreover, a quantitative analysis of fluoromicroscopic images of tumor necrosis at $24 \mathrm{~h}$ after injection showed differences in normalized fluorescence intensities between hypericin and labeled lipoproteins of 50-100\%, reflecting a shifted pattern in localization. We conclude that our results are indicative of a release of hypericin from the lipoprotein complex at some point along its way through the peri-necrotic tumor area and the necrotic tissue debris, which is in line with the hypothesis of a compound-specific mechanism.
\end{abstract}

\section{Introduction}

As a consequence of their high specificity and sensitivity, necrosis avid contrast agents (NACAs) may be used for noninvasive 'hot-spot imaging' and localization of necrotic tissue

Correspondence to: Dr P.A.M. De Witte, Laboratorium voor Farmaceutische Biologie, Faculteit Farmaceutische Wetenschappen, KU Leuven, O and N II Herestraat 49 - bus 824, 3000 Leuven, Belgium

E-mail: peter.dewitte@pharm.kuleuven.be

Key words: hypericin, necrosis, necrosis avid contrast agents, mechanism, lipoproteins, tumor in the diagnosis and therapy follow-up of numerous disorders. Thus far, these compounds have mainly been investigated as markers for magnetic resonance imaging (MRI) of ischemic myocardial injury (1-4), pancreatitis, brain infarct and therapeutic evaluation after interventional therapies (5). Moreover, NACAs may fulfill several applications in the field of oncology. Due to the known prevalence of spontaneous necrosis in bulky human tumors $(30-80 \%$ of the tumor mass) (6-8), non-invasive methods of assessing the presence and extent of necrosis may result in a better tumor staging and tailoring of treatment to the needs of the individual patient. On the other hand, a non-invasive evaluation of the necrotic tumor fraction after necrosis-inducing therapies, such as radiofrequency ablation and intratumoral ethanol injection, may be a valuable method for the assessment of treatment efficacy (5). In this way, the detection of incomplete tumor ablation may allow early interventions to avoid the risk of tumor re-growth. So far, non-invasive methods of determining the amount of necrosis (CT, MRI) are based on non-specific measurements, such as differences in tissue perfusion and the apparent diffusion coefficient of water $(9,10)$. However, during the first months following ablative treatments, all current imaging methods suffer from non-specific enhancement in the periphery of the ablation, which confuses residual tumor with the benign hyperemic and regenerative tissues $(11,12)$.

Hypericin, a naturally occurring photosensitizer derived from the plant genus Hypericum, was recognized as a nonporphyrin NACA, as the compound showed a peculiar affinity for both necrotic- and irreversibly-damaged ischemic tissue. Recently, radiolabeled derivatives of hypericin ([ $\left.{ }^{123} \mathrm{I}\right]-$ iodohypericin) have been extensively studied in hepatic and myocardial infarcted animal models (13). For instance, after a systemic injection it was found that $\left[{ }^{123} \mathrm{I}\right]$-iodohypericin concentrated in infarcted tissue (liver and myocardium) $>10$ - to 18 -fold in normal surrounding tissues (13). A similar affinity was found in bulky subcutaneous tumors consisting of large areas of spontaneous necrosis, where compared to viable tumors a $>19$-fold activity of [ $\left.{ }^{123} \mathrm{I}\right]$-iodohypericin was recorded (unpublished data). Such promising results suggest possible applications for hypericin derivatives in the field of clinical diagnosis of ischemic, infarcted tissues and bulky necrotic tumors. Nonetheless, despite multiple investigations 
to exploit necrosis avidity for medical purposes, very little is known about the underlying mechanism. A more complete insight into this mechanism is important in view of the future chemical development of new NACAs with improved target to non-target ratios and minimal toxicity. In an earlier study by Hofmann and coworkers (14), the authors attributed the necrosis avidity of the NACA gadophrin-2 to a non-specific mechanism of trapping in necrotic areas due to albuminbinding. To verify whether this 'trapping' mechanism is also valid for hypericin, which is mainly bound to lipoproteins in the blood, it was of interest to define the role of lipoproteins as regards the uptake and retention of hypericin in necrotic tumor areas. For this purpose, hypericin was traced in necrotic subcutaneous tumors in mice as a function of time, and a colocalization with labeled lipoproteins was explored in viable tumor areas and necrotic tumor regions. In addition, intratumoral localization of a high molecular weight FITCdextran (MW 2.106) was used to assess tumor blood perfusion and extravasation in peri-necrotic areas.

\section{Materials and methods}

Animals and the tumor system. Radiation-induced fibrosarcoma (RIF-1) tumor cells $\left(2.10^{6}\right)$ were inoculated subcutaneously on the lower dorsum of the female $\mathrm{C}_{3 \mathrm{H}} / \mathrm{Km}$ mice (weight range 21-25 g, purchased from Charles River Laboratories, France). Mice were used when the tumor volume reached $0.5 \mathrm{~cm}^{3}$. All aspects of the animal experiment and husbandry were carried out in compliance with national and European regulations and were approved by the Animal Care and Use Committee of our institute.

Preparation and administration of the compounds. The antivascular agent 5,6-dimethylxanthenone-4-acetic acid (DMXAA, obtained from Sigma, St. Louis, MO, USA) was used to induce uniform tumor necrosis (15). All mice received an i.p. injection $(15 \mathrm{mg} / \mathrm{kg})$ with DMXAA $24 \mathrm{~h}$ before a coinjection of hypericin and $\mathrm{DiOC}_{18}$, or hypericin and FITCdextran. Prior to injection, DMXAA was dissolved in phosphate-buffered saline $(1 \mathrm{mg} / \mathrm{ml})$.

Hypericin was synthesized from emodin anthraquinone according to Falk et al (16) and dissolved prior to injection in a mixture of 25\% dimethylsulfoxide (DMSO), 25\% polyethylene glycol (PEG) 400 and water $(2 \mathrm{mg} / \mathrm{ml})$. Hypericin $(7 \mathrm{mg} / \mathrm{kg}$ ) was i.v. administered, immediately followed by an i.v. injection of FITC-dextran (fluorescein isothiocyanate dextran, $\mathrm{M}_{\mathrm{r}} 2.10^{6}$, obtained from Sigma, St. Louis, MO, USA) (300 mg/kg, in PBS $30 \mathrm{mg} / \mathrm{ml}$ ) in DMXAA-treated RIF-1 tumor-bearing animals. The mice were sacrificed $5 \mathrm{~min}, 1 \mathrm{~h}$ or $24 \mathrm{~h}$ after injection.

In another set of experiments, hypericin was simultaneously i.v. administered $(7 \mathrm{mg} / \mathrm{kg})$ with $\mathrm{DiOC}_{18}\left(3,3^{\prime}\right.$-dioctadecyloxacarbocyanine, Molecular Probes Inc., Eugene, OR, USA) ( $7 \mathrm{mg} / \mathrm{kg})$ to DMXAA-treated, RIF-1 tumor-bearing animals $5 \mathrm{~min}, 2 \mathrm{~h}, 4 \mathrm{~h}, 15 \mathrm{~h}$ or $24 \mathrm{~h}$ before sacrifice. $\mathrm{DiOC}_{18}$ was suspended $(2 \mathrm{mg} / \mathrm{ml})$ in $40 \%$ propylene glycol, $10 \%$ ethanol, $4 \%$ Tween- 80 and water, after which the mixture was sonicated at $60^{\circ} \mathrm{C}$ for $2 \mathrm{~h}$.

Intratumoral localization of hypericin, DiOC ${ }_{18}$ and FITCdextran. Tumor samples were harvested, immediately mounted in medium (Tissue Tek-embedding medium, Miles Inc., Elkhart, IN, USA) and immersed in liquid nitrogen. Different cryostat sections $(5 \mu \mathrm{m})$ were cut at mid-tumor, where the tumor mass was at its largest. Sections were first investigated by means of fluorescence microscopic imaging (Axioskop 2 plus, Carl Zeiss, Göttingen, Germany), to obtain a composite image of hypericin with either the FITC-dextran or $\mathrm{DiOC}_{18}$-labeled lipoprotein distribution. To specifically visualize hypericin, the Zeiss filter set 14 (ex: BP 510-560 nm, em: LP $590 \mathrm{~nm}$ ) was used, whereas FITC-dextran and DiOC $_{18}$ were examined with the Zeiss filter set 10 (ex: BP 450-490 $\mathrm{nm}$, em: BP 515-565 nm). Subsequently, the same tissue sections were stained with hematoxylin and eosin (H\&E). Fluorescence images were acquired using a light-sensitive charge-coupled device digital camera (AxioCam HR, Carl Zeiss).

A KS imaging software system (Carl Zeiss, Vision, Hallbergmoos, Germany) was used for the quantification of overlay fluorescence images. Overlay images of either necrotic tumor regions $(n=15)$, or viable tumor areas $(n=15)$, taken throughout 3 different tumors at 24 h p.i., were analyzed. A quantitative analysis was performed as before (17), by subdividing the images in 2269 square fields of $38.7 \mu \mathrm{m}^{2}$ (24 pixels/field) and by measuring the average fluorescence intensity per field for hypericin and $\mathrm{DiOC}_{18}$, respectively. The data were normalized for the maximal fluorescence intensity of each compound and expressed as a percentage fluorescence intensity (\% f.i.). From these field-by-field data, scattergrams were constructed with axes representing the percentage fluorescence intensity of each compound. In addition, the absolute field-by-field differences in fluorescence intensity for hypericin and $\mathrm{DiOC}_{18}$ [i.e. $\Delta=\mid(\%$ f.i. .hypericin $-\%$ f.i. DiOC18 $\mid$ |] were calculated. The differences were grouped in fractions with increments of $10 \%$ and the percentage of fields corresponding to each of the fractions determined. Statistical analysis was performed using the two-way ANOVA with Bonferroni test, Prism 4.00, GraphPad Software, San Diego, CA, USA.

\section{Results}

DMXAA-treated tumors. Upon histological evaluation, tumors from DMXAA-treated animals were characterized by geographically scattered tumor necrosis and hemorrhage, as described in the literature $(15,18)$. Histologically, tumors were clearly divided into three different regions, i.e. necrotic areas, viable regions and peri-necrotic areas (defined as the transitional zone between viable tumor areas and necrotic regions). Viable tumor regions consisted of viable sarcoma cells with numerous mitoses and evident vasculature. Hemorrhagic necrotic zones were formed due to the massive extravasation of erythrocytes and subsequent cessation of blood flow (19). The peri-necrotic areas were typically characterized by inflamed tumor blood vessels, due to their swollen, congested appearance and thickened endothelial wall on the H\&E stains. Interstitial bleeding was typically found in peri-necrotic areas.

Intratumoral localization of hypericin and FITC-dextran. To visualize tumor perfusion and the leakiness of tumor blood 


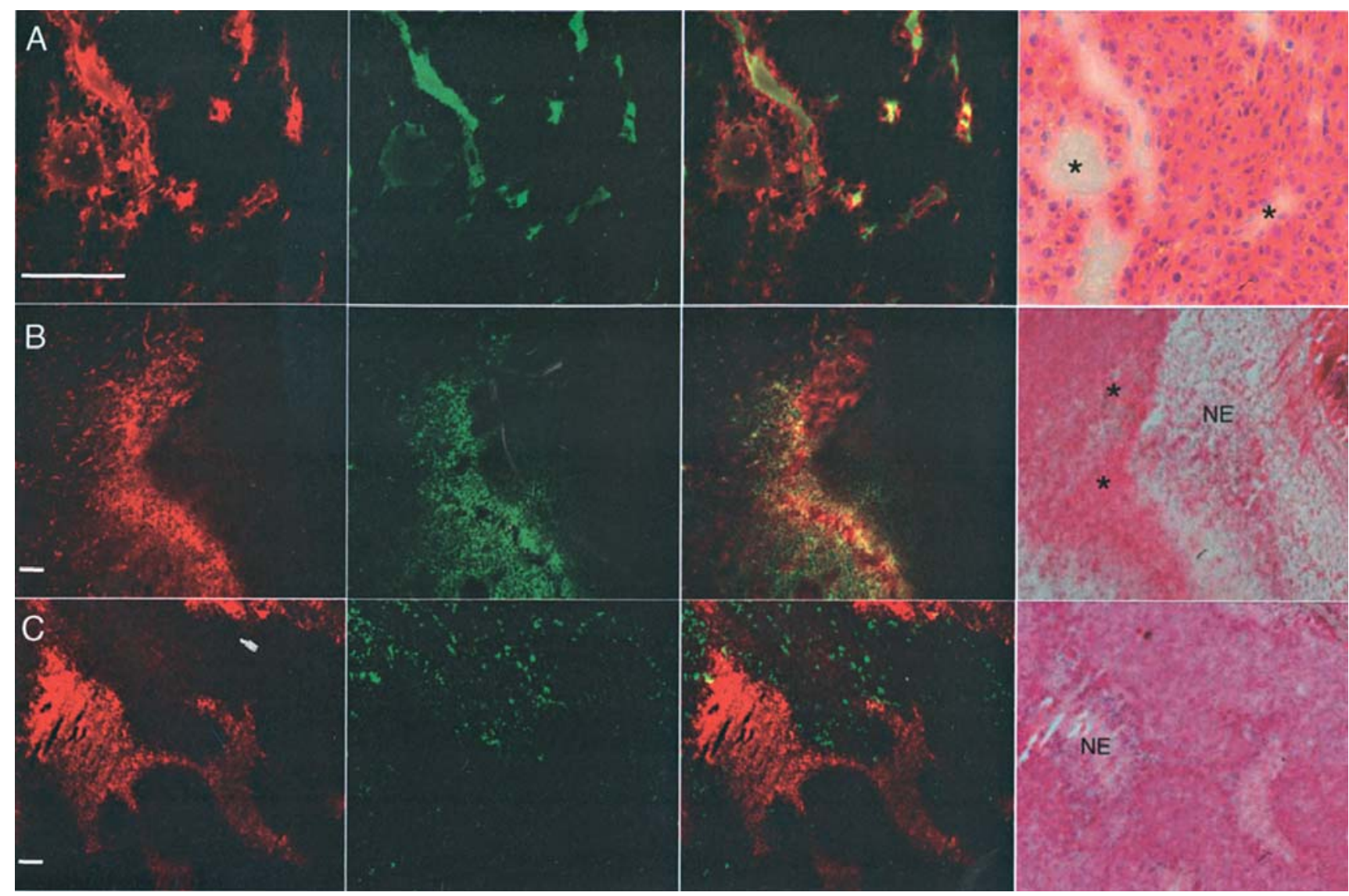

Figure 1. Fluorescence photomicrographs of 5- $\mu \mathrm{m}$ RIF-1 mouse tumor sections sampled at $5 \mathrm{~min}(\mathrm{~A}), 1 \mathrm{~h}(\mathrm{~B})$ and $24 \mathrm{~h}$ (C) after an i.v. injection of $7 \mathrm{mg} / \mathrm{kg}$ hypericin (red fluorescence, first column) and $300 \mathrm{mg} / \mathrm{kg}$ FITC-dextran (green fluorescence, second column). Overlay pictures are depicted in the third column. In the right column the corresponding H\&E stains are shown. Representative images are shown. NE, necrotic tumor; ${ }^{*}$ inflamed blood vessel(s); scale bar, $100 \mu \mathrm{m}$.

vessels, a macromolecular green fluorescent FITC-dextran was co-injected with hypericin. At 5 min after injection (Fig. 1A), both FITC-dextran (green fluorescence) and hypericin (red fluorescence) were confined to the lumen of inflamed blood vessels in peri-necrotic areas. Unlike FITCdextran, hypericin was also apparent in the endothelial vessel wall. At $1 \mathrm{~h}$ after injection (Fig. 1B), the macromolecular FITC-dextran and hypericin extravasated from hyperpermeable inflamed vessels in peri-necrotic regions, in contrast to the viable tumor areas where both tracers were still mainly confined to the vessel lumen (not shown). At $24 \mathrm{~h}$ after injection, the extravasation of hypericin and FITC-dextran was complete and an inverse relationship in the distribution was observed (Fig. 1C). Though still present in the viable tumor, hypericin accumulated in necrotic tumor areas to much higher levels, whereas the green fluorescence of FITC-dextran formed a patchy pattern throughout the peri-necrotic areas and viable tumor, showing no affinity for the necrotic tumor.

Intratumoral localization of hypericin and DiOC 18 . Bloodborne hypericin associates mainly with HDL (high-density lipoproteins) and LDL (low-density lipoproteins) $(20,21)$. It was therefore of interest to verify whether the uptake and retention of hypericin in the necrotic tumor was facilitated by non-specific lipoprotein transport or alternatively by relocation and the specific binding of hypericin after dissociation from the lipoprotein complex. For that purpose, $\mathrm{DiOC}_{18}$ was simultaneously injected with hypericin into the bloodstream. $\mathrm{DiOC}_{18}$ is a green fluorescent analogue of DiIC 18 (22), a marker that by means of its very lipophilic $\mathrm{C}_{18}$ moieties avidly binds to lipoproteins without altering their affinity for the receptors. The compound has already been used before to track down the migration of lipoproteins in tumor tissue (17). Fluorescence microscopic analysis of necrotic and perinecrotic regions in tumors showed a time-dependent shifting in the localization of hypericin and labeled lipoproteins. At 5 min after injection, the two compounds were confined to the tumoral blood vessels in the peri-necrotic tumor areas (Fig. 2A). After $2 \mathrm{~h}$, substantial amounts of hypericin and $\mathrm{DiOC}_{18}$-labeled lipoproteins leaked from the hyperpermeable inflamed vessels into the peri-necrotic areas (Fig. 2B). At $4 \mathrm{~h}$ after administration (Fig. 2C), the images showed hypericin and $\mathrm{DiOC}_{18}$-labeled lipoproteins traversing the peri-necrotic areas and penetrating the necrotic zones. Contrary to the labeled lipoproteins, at this time/point hypericin started to discriminate between necrotic zones and peri-necrotic or viable tumor areas. As a matter of fact, $15 \mathrm{~h}$ after injection the distribution pattern of hypericin appeared almost inverse to that observed for lipoproteins (Fig. 2D). At 24 h, $\mathrm{DiOC}_{18}$ green fluorescence was cleared from the necrotic areas, resulting in a striking 


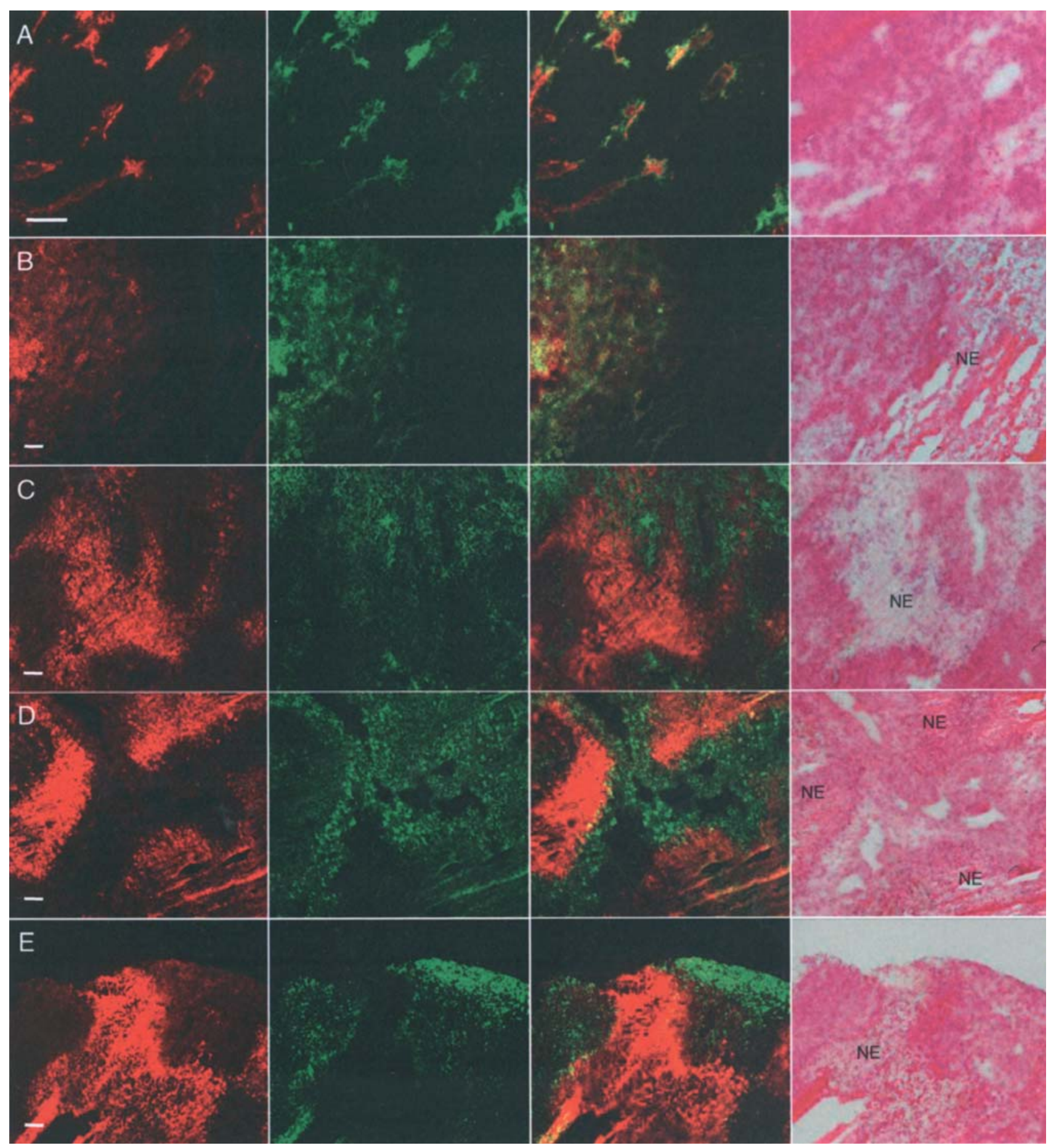

Figure 2. Fluorescence photomicrographs of 5- $\mu \mathrm{m}$ RIF-1 mouse tumor sections sampled at $5 \mathrm{~min}(\mathrm{~A}), 2 \mathrm{~h} \mathrm{(B),} 4 \mathrm{~h}(\mathrm{C}), 15 \mathrm{~h}$ (D) and $24 \mathrm{~h}$ (E) after an i.v. injection of $7 \mathrm{mg} / \mathrm{kg}$ hypericin (red fluorescence, first column) and $7 \mathrm{mg} / \mathrm{kg} \mathrm{DiOC}{ }_{18}$ (green fluorescence, second column). Overlay pictures are depicted in the third column. In the right column the corresponding H\&E stains are shown. Representative images are shown. NE, necrosis; scale bar, $100 \mu \mathrm{m}$.

difference in distribution between hypericin and $\mathrm{DiOC}_{18^{-}}$ labeled lipoproteins (Fig. 2E). Whereas hypericin concentrated preferentially on the necrotic tumor, the $\mathrm{DiOC}_{18}$-labeled lipoproteins obviously showed no affinity for necrotic debris.

Quantitative measurements of the colocalization of hypericin and $\mathrm{DiOC}_{18}$-labeled lipoproteins at $24 \mathrm{~h}$ after injection were examined by a field-by-field analysis of fluorescent overlay images of either viable tumor areas or necrotic tumor regions. In comparison to viable tumor areas, scattergrams (Fig. 3) of necrotic tumor regions shifted towards a relatively higher $\%$ f.i. of hypericin but a lower $\%$ f.i. of $\mathrm{DiOC}_{18}$-labeled lipoproteins. The percentage of fields that fell within the fractions of grouped absolute differences $(\Delta)$ was scored for the necrotic and viable tumor regions (Fig. 4). In the viable tumor areas, the majority of fields $(52.7 \%)$ scored $\Delta$ values of $<20 \%$, with only $3.9 \%$ fields in the $\Delta$ range of $50 \%$ or higher. In the necrotic tumor regions, $79.3 \%$ of fields were recorded between $\Delta(50-100 \%)$, with only $5.8 \%$ fields 

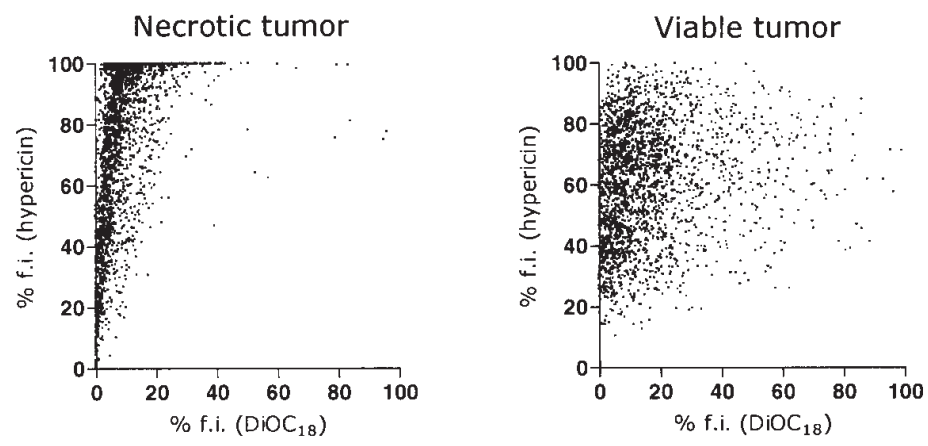

Figure 3. Quantification results of fluoromicroscopic images of necrotic tumor areas and viable tumor regions taken at $24 \mathrm{~h}$ after coadministration of hypericin and $\mathrm{DiOC}_{18}$. Scattergrams represent 2269 field-by-field relationships between normalized fluorescence intensities (\% f.i.) of hypericin and DiOC 18 . Results of representative fluorescent overlay images are shown.

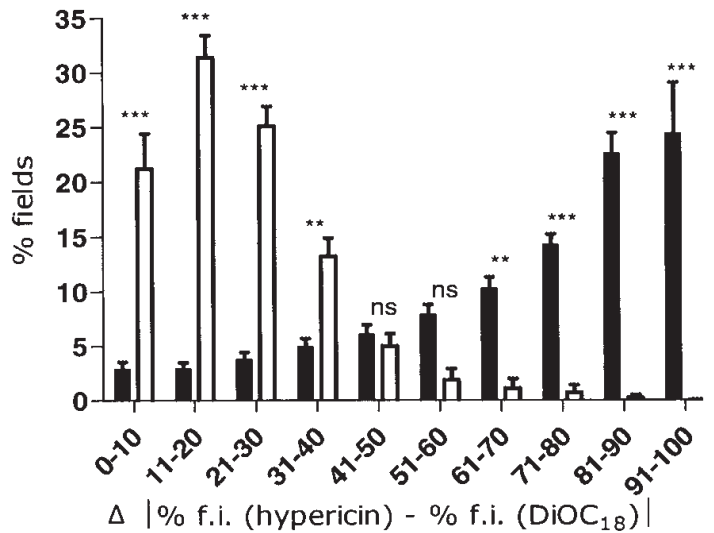

Figure 4. Distribution of percentages of fields as a function of absolute differences $(\Delta)$ between normalized fluorescence intensities (\% f.i.) of hypericin and $\mathrm{DiOC}_{18}$ in viable (white bars) and necrotic (black bars) tumor regions. $\Delta$ values were grouped in fractions with increments of $10 \%$. Data are expressed as mean \pm SD (average of the analysis of three tumors and fifteen fluorescent overlay images per viable or necrotic tumor region); ${ }^{* * *} \mathrm{P}<0.001,{ }^{* *} \mathrm{P}<0.01 ; \mathrm{ns}$, not significant.

having relatively small differences in the range $0-20 \%$. In other words, differences in fluorescence intensity between hypericin and $\mathrm{DiOC}_{18}$ were markedly more pronounced in the necrotic tumor areas as compared to the viable tumor regions.

\section{Discussion}

To tackle the issue of whether a compound-specific or a nonspecific mechanism based on plasma protein transport is involved in the preferential accumulation of hypericin in the necrotic tumor areas, we performed a colocalization study comparing the intratumoral migration of hypericin and labeled lipoproteins. Tumors were used before the onset of spontaneous necrosis, since in general the development of necrotic areas in tumor tissue is highly variable, even within tumors of the same strain and origin. To obtain necrotic tumors in a reproducible way, animals were pre-treated with the antivascular agent DMXAA, inducing within $24 \mathrm{~h}$ a geographically scattered tumor necrosis and hemorrhage $(15,18)$.

To demonstrate the extravasation of blood-borne molecules and proteins in peri-necrotic regions, the distribution of high molecular weight FITC-dextran was followed throughout the tumor at different time intervals after injection. As a rule, the extravasation and interstitial penetration of FITC-dextran is typically restricted due to its high molecular weight and size (23). However, as DMXAA attacks and partially breaks down the endothelial lining, the vasculature becomes very leaky and consequently is permeable even to high molecular weight compounds. The injection of hypericin and $\mathrm{DiOC}_{18}$ resulted in a similar swift extravasation pattern, permitting hypericin and labeled lipoproteins to readily reach the necrotic tumor areas. Whereas hypericin already showed clear necrosis avid characteristics $4 \mathrm{~h}$ after injection, a similar selective accumulation in the necrotic tumor was not demonstrated either for the $\mathrm{DiOC}_{18}$-labeled lipoproteins or FITC-dextran. With regard to the latter, a penetration into the necrotic debris did occur, although within $24 \mathrm{~h}$ the tissue was cleared, apparently due to a lack of any affinity of the compound for necrotic debris. These results suggest a dissociation of hypericin from the lipoprotein complex, which is in line with an earlier finding concerning viable nonnecrotic tumors, where a similar release of hypericin from lipoproteins was demonstrated in the viable tumor mass (17).

In line with this outcome, a quantitative analysis of the fluoromicroscopic images of necrotic tumor at $24 \mathrm{~h}$ showed substantial differences in normalized fluorescence intensity regarding localization between hypericin and lipoproteins. These results once more strongly suggest a release of hypericin from the lipoprotein complex at some point along its way throughout the peri-necrotic tumor area and the necrotic debris. The fact that lipoprotein transport is not a prerequisite for the uptake and retention of hypericin in necrotic tumor tissue, supports the hypothesis of a compound-specific mechanism, as opposed to the albumin-related trapping mechanism proposed by Hofmann and coworkers (14). However, the latter mechanism has been criticized before (24), since many agents with a high degree of serum albumin binding do not act as NACAs. The present results therefore are in line with the concept that albumin or lipoprotein binding is not the mechanism underlying necrosis avidity, but only a parallel and non-specific feature shared by both NACAs and non-NACA substances (13).

The behavior of hypericin and other NACAs, showing a high affinity for necrosis, independent from the cell type or 
cause of injury (13), resembles the response of immune cells well when challenged with necrotic tissue. In contrast to apoptosis, necrotic cell death is typically associated with a loss of membrane integrity and inflammation. A number of studies on necrosis, investigating the identity of stimuli that activate immune cells, have shown that necrotic cell death activates the immune system by the release of heat shock proteins and degraded cellular peptides (25-27). This primitive mechanism of immune response to cellular disintegration upon necrotic cell death is considered as an autonomous healing process of the living organisms. Hypothetically, hypericin may specifically associate to such degraded proteins or peptides present in the necrotic milieu. The delayed clearance of hypericin is probably related to the progressive infiltration and phagocytosis by inflammatory cells, which is a timeconsuming process. Presumably, after being engulfed by immune cells, hypericin is removed together with necrotic debris and replaced by granulation tissue. Although attractive, it definitely remains to be investigated whether this hypothesis is sound and whether the general mechanism is an important clue in the elucidation of the mechanism underlying the necrosis-avid characteristics of hypericin and other NACAs.

In conclusion, the present study is indicative of a release of hypericin from the lipoprotein complex at some point along its way through the peri-necrotic tumor area and the necrotic tissue debris. This finding is in line with the idea of a compound-specific mechanism. It is presently unknown to which specific constituents hypericin tightly binds in the necrotic space. Further investigations are needed to elucidate the specific interactions between hypericin and the necrotic milieu.

\section{Acknowledgements}

This work was supported by grants awarded by Fonds voor Wetenschappelijk Onderzoek-Vlaanderen (FWO Vlaanderen) and a Geconcerteerde Onderzoeksactie (GOA) of the Flemish Government.

\section{References}

1. Ni Y, Pislaru C, Bosmans H, Pislaru S, Miao Y, Van de Werf F, Semmler W and Marchal G: Validation of intracoronary delivery of metalloporphyrin as an in vivo "histochemical staining' for myocardial infarction with MR imaging. Acad Radiol 5: S37-S41, 1998.

2. Pislaru S, Ni Y, Pislaru C, Bosmans H, Miao Y, Bogaert J, Dymarkowski S, Semmler W, Marchal G and Van de Werf F: Noninvasive measurements of infarct size after thrombolysis with a necrosis-avid MRI contrast agent. Circulation 99: 690-696, 1999 .

3. Choi S, Choi S, Kim S, Lim K, Lim C, Gong G, Kim H, Weinmann $\mathrm{H}$ and Lim T: Irreversibly damaged myocardium at MR imaging with a necrotic tissue-specific contrast agent in a cat model. Radiology 215: 863-868, 2000.

4. Jin JY, Teng GJ, Feng Y, Wu YP, Jin QD, Wang Y, Wang Z, Lu Q, Jiang YB, Wang SQ, Chen F, Marchal G and Ni YC: MR imaging of acute reperfused myocardial infarction: intraindividual comparison of ECIII-60 and Gd-DTPA in a swine model. CVIR 30: 248-256, 2007.

5. Ni Y, Chen F, Mulier S, Sun X, Yu J, Landuyt W, Marchal G and Verbruggen A: Magnetic resonance imaging after radiofrequency ablation in a rodent model of liver tumor: tissue characterization using a novel necrosis avid contrast agent. Eur Radiol 16: 1031-1040, 2006.

6. Cooper E, Bedford A and Kenny T: Cell death in normal and malignant tissues. Adv Cancer Res 21: 59-120, 1975.
7. Koljenovic S, Choo-Smith L, Bakker Schut T, Kros J, van den Berge H and Puppels G: Discriminating vital tumor from necrotic tissue in human glioblastoma tissue samples by Raman spectroscopy. Lab Invest 82: 1265-1277, 2002.

8. Tufto I and Rofstad E: Interstitial fluid pressure, fraction of necrotic tumor tissue, and tumor cell density in human melanoma xenografts. Acta Oncol 37: 291-297, 1998.

9. Silva A, Hara A, Leighton J and Heppell J: CT colonography with intravenous contrast material: varied appearances of colorectal carcinoma. Radiographics 25: 1321-1334, 2005.

10. Lyng H, Haraldseth $\mathrm{O}$ and Rofstad E: Measurement of cell density and necrotic fraction in human melanoma xenografts by diffusion weighted magnetic resonance imaging. Magn Reson Med 43: 828-836, 2000.

11. Goldberg S: Science to practice: can we differentiate residual untreated tumor from tissue responses to heat following thermal tumor ablation? Radiology 234: 317-318, 2005.

12. Guan Y, Sun L, Zhou X, Li X and Zheng X: Hepatocellular carcinoma treated with interventional procedures: CT and MRI follow-up. World J Gastroenterol 10: 3543-3548, 2004.

13. Ni Y, Huyghe D, Verbeke K, de Witte PA, Nuyts J, Mortelmans L, Chen F, Marchal G, Verbruggen A and Bormans G: First preclinical evaluation of mono- $\left[{ }^{123} \mathrm{I}\right]$ iodohypericin as a necrosis-avid tracer agent. Eur J Nucl Med Mol Imaging 33: 595-601, 2006.

14. Hofmann B, Bogdanov A, Marecos E, Ebert W, Semmler W and Weissleder R: Mechanism of gadophrin-2 accumulation in tumor necrosis. J Magn Reson Imaging 9: 336-341, 1999.

15. Ching L, Goldsmith D, Joseph W, Korner H, Sedgwick J and Baguley B: Induction of intratumoral tumor necrosis factor (TNF) synthesis and hemorrhagic necrosis by 5,6-dimethylxanthenone-4-acetic acid (DMXAA) in TNF knockout mice. Cancer Res 59: 3304-3307, 1999.

16. Falk H, Meyer J and Oberreiter M: A convenient semisynthetic route to hypericin. Monatsh Chem 124: 339-341, 1993.

17. Van de Putte M, Roskams T, Vandenheede J, Agostinis P and de Witte P: Elucidation of the tumoritropic principle of hypericin. Br J Cancer 92: 1406-1413, 2005.

18. Liu J, Ching L, Goldthorpe M, Sutherland R, Baguley B, Kirker J and McKeage M: Antitumour action of 5,6-dimethylxanthenone4 -acetic acid in rats bearing chemically induced primary mammary tumours. Cancer Chemother Pharmacol 59: 661-669, 2007.

19. Baguley B: Antivascular therapy of cancer: DMXAA. Lancet Oncol 4: 141-148, 2003.

20. Lavie G, Mazur Y, Lavie D, Prince A, Pascual D, Liebes L, Levin B and Meruelo D: Hypericin as an inactivator of infectious viruses in blood components. Transfusion 35: 392-400, 1995.

21. Chen B, Xu Y, Roskams T, Delaey E, Agostinis P, Vandenheede $\mathrm{J}$ and de Witte P: Efficacy of antitumoral photodynamic therapy with hypericin: relationship between biodistribution and photodynamic effects in the RIF-1 mouse tumor model. Int J Cancer 93: 275-282, 2001.

22. Pitas R, Innerarity T, Weinstein J and Mahley R: Acetoacetylated lipoproteins used to distinguish fibroblasts from macrophages in vitro by fluorescence microscopy. Arteriosclerosis 1: 177-185, 1981 .

23. Dreher M, Liu W, Michelich C, Dewhirst M, Yuan F and Chilkoti A: Tumor vascular permeability, accumulation, and penetration of macromolecular drug carriers. J Natl Cancer Inst 98: 335-344, 2006.

24. Ni Y, Adzamli K, Miao Y, Cresens E, Yu J, Periasamy M, Adams $M$ and Marchal G: MRI contrast enhancement of necrosis by MP-2269 and gadophrin-2 in a rat model of liver infarction. Invest Radiol 36: 97-103, 2001.

25. Basu S, Binder R, Suto R, Anderson K and Srivastava P: Necrotic but not apoptotic cell death releases heat shock proteins, which deliver a partial maturation signal to dendritic cells and activate the NF-kappa B pathway. Int Immunol 12: 1539-1546, 2000.

26. Sauter B, Albert M, Francisco L, Larsson M, Somersan S and Bhardwaj N: Consequences of cell death: exposure to necrotic tumor cells, but not primary tissue cells or apoptotic cells, induces the maturation of immunostimulatory dendritic cells. J Exp Med 191: 423-434, 2000.

27. Srivastava P: Interaction of heat shock proteins with peptides and antigen presenting cells: chaperoning of the innate and adaptive immune responses. Annu Rev Immunol 20: 395-425, 2002 . 